Discussion GEQUAS enables proficiency testing for the most biomonitoring parameters which are used for the exposure assessment at workplaces (metals, solvents, pesticides, new emerging chemicals). Levels and specification are carefully adjusted to the practice.

\section{MIXIE, A TOOL TO IMPROVE ASSESSMENT OF CHEMICAL RISK IN CASE OF MULTIPLE EXPOSURE}

L Coates*, N Bertrand, S Binet, P Campo, F Clerc, B La Rocca, F Pillière. National Research and Safety Institute for the Prevention of Occupational Accidents and Diseases (INRS)

\subsection{6/oemed-2018-ICOHabstracts.1131}

Introduction Multiple exposure to chemicals is a common situation in workplaces. However, most methods used to evaluate chemical risk do not consider the potential effects of mixtures. The aim is to present a tool helping to evaluate chemical risk in case of multiple exposure.

Methods MiXie is a web tool (http://www.inrs-mixie.fr/http:// www.inrs-mixie.fr/) developed in Quebec in 1997 and adapted to the French context by the French National Research and Safety Institute for the prevention of occupational accidents and diseases (INRS). It helps industrial hygienists to assess the potential risk of multi-exposure. Additivity of effects is the basic assumption.

Results Whenever measurements of atmospheric concentrations are provided, MiXie calculates the exposure index of the mixture (i.e. the sum of the ratios between each concentration measured and its occupational exposure limit value $x$ 100). If this index exceeds $100 \%$, MiXie signals that there is a potential risk for certain organs, even though each limit value is respected.

When measurements of atmospheric concentrations are not provided, MiXie highlights the common effects classes of the substances present and warns about a potential risk of additive effects.

If the mixture contains a substance belonging to the 'cancer' or 'sensitizer' effect class, additivity does not apply and MiXie warns the industrial hygienist regardless of the concentration measured.

Conclusion The MiXie database helps to identify potential risk situations related to multi-exposure to chemicals. Such situations may go unnoticed with a monosubstance approach. But MiXie users should be aware of its limits: additivity does not apply to all situations, the number of substances is restricted (130), etc. Experts are working to improve the tool by increasing the number of substances, making it more userfriendly, etc.

\section{EFFECTS OF METAL-RICH PARTICULATE MATTER EXPOSURE ON EPSTEIN-BARR VIRUS AND HUMAN ENDOGENOUS RETROVIRUS W (HERV-W) METHYLATION HEALTHY STEEL-WORKERS}

\footnotetext{
1,2V Bollati ${ }^{*},{ }^{1,2} \mathrm{M}$ Bonzini, ${ }^{1} \mathrm{R}$ Mercorio, 'L Angelici, ${ }^{1} \mathrm{~J}$ Mariani, 'S Delbue, ${ }^{4} \mathrm{P}$ Apostoli,

1,2 AC Pesatori. 'EPIGET Lab - Dept. Clinical Sciences and Community Health, Università degli Studi di Milano, Milan, Italy; ${ }^{2}$ Fondazione IRCCS Ca' Granda Ospedale Maggiore Policlinico, Occupational Medicine Unit, Milan, Italy; ${ }^{3}$ Dept Biomedical, Surgical and Dental Sciences, Università degli Studi di Milano, Milan, Italy; ${ }^{4}$ Dept Experimental and Applied Medicine, University of Brescia, Brescia, Italy
}

10.1136/oemed-2018-ICOHabstracts.1132
Introduction Inhaled particulate pollutants have been shown to produce systemic changes in DNA methylation. Global hypomethylation has been associated to viral sequence reactivation, possibly linked to the activation of pro-inflammatory pathways occurring after exposure. We aimed at evaluating the effects of PM exposure on DNA methylation of the Wp promoter of the Epstein-Barr Virus (EBV-Wp) and the promoter of the human-endogenous-retrovirus w (HERV-w), chosen as a paradigm of an exogenous virus and an endogenous retroviral sequence, in workers in an electric furnace steel plant with well-characterised exposure to metal-rich particulate matter.

Methods We measured EBV-Wp and HERV-w DNA methylation through bisulfite PCR Pyrosequencing on peripheral blood leukocytes DNA obtained from 63 male healthy workers, on the first day of a work week (baseline, after 2 days off work) and after 3 days of work (post-exposure). We determined individual exposure to inhalable particles and metals for all subjects. Paired t-test was used to compare baseline and post-exposure samples. Linear mixed models were fitted to evaluate the association between metal-rich particle exposure and DNA methylation.

Results Comparing samples obtained at baseline and after 3 days of work, the mean methylation of EBV-Wp was significantly higher at baseline compared to post-exposure (baseline $=56.7$; postexposure $=47.9 ; \mathrm{p}$-value $=0.009$ ), whereas the mean methylation of HERV-w did not significantly differ. In a regression model adjusted for age, body mass index and smoking, nickel, arsenic and lead had a positive association with EBV-Wp methylation (nickel: $\beta=16.16, \quad$ p-value $<0.001$; arsenic: $\quad \beta=13.0, \quad p$-value $=0.02$; lead: $\beta=16.53$, $p$ value $<0.001)$.

Conclusions The difference observed comparing baseline and postexposure samples may be suggestive of a rapid change in EBV methylation induced by air particles, while correlation between EBV methylation and metal exposure may represent an adaptive mechanism that should be further characterised in future investigations.

\section{STEROID HORMONES, MELATONIN AND VITAMIN D IN FEMALE HOSPITAL NURSES WORKING WITH '1-1-1' RAPID CYCLE SHIFT}

C Maggioni, E Crespi, E Polledri, R Mercadante, G Costa, S Fustinoni ${ }^{*}$. Dipartimento di Scienze Cliniche e di Comunità, Università degli Studi di Milano e Fondazione IRCCS Ca' Granda Ospedale Maggiore Policlinico, Via S. Barnaba, 8- 20122 Milano, Italia

\subsection{6/oemed-2018-ICOHabstracts.1133}

Introduction Shiftwork that disrupts circadian rhythms has been classified as probable carcinogenic to humans by IARC (2007). Among possible mechanisms of this effect, the modification of hormone homeostasis has been advocated. Aim of the present study was to evaluate the influence of night-shift work on the levels of steroid hormones, melatonin, and serum vitamin $\mathrm{D}$ in hospital female nurses.

Methods Ninety-five female hospital nurses were recruited: 45 performing ' $1-1-1$ ' fast rotating shift schedule on 5 day cycle (morning - afternoon - night - rest - rest) and 50 working only on dayshift, as controls. Specimens were collected to measure 13 serum steroid hormones, salivary cortisol, cortisone and melatonin in morning and evening samples, and serum vitamin D. All markers were assayed by liquid chromatography coupled with triple quadrupole mass spectrometry. 